\title{
On Scaling Laws and Alfvénic Magnetic Fluctuations in Molecular Clouds
}

\author{
Taoling Xie \\ Laboratory for Millimeter-wave Astronomy, Department of Astronomy, University of \\ Maryland, College Park, MD 20742; email: tao@astro.umd.edu
}

To appear in Astrophysical Journal Letters

\begin{abstract}
Under the basic assumption that the observed turbulent motions in molecular clouds are Alfvénic waves or turbulence, we emphasize that the Doppler broadening of molecular line profiles directly measures the velocity amplitudes of the waves instead of the Alfvén velocity. Assuming an equipartition between the kinetic energy and the Alfvénic magnetic energy, we further propose the hypothesis that observed standard scaling laws in molecular clouds imply a roughly scale-independent fluctuating magnetic field, which might be understood as a result of strong wave-wave interactions and subsequent energy cascade. We predict that $\sigma_{v} \propto \rho^{-0.5}$ is a more basic and robust relation in that it may approximately hold in any regions where the spatial energy density distribution is primarily determined by wave-wave interactions, including gravitationally unbound regions. We also discuss the fact that a scale-independent $\sigma_{B}^{2}$ appears to contradict existing 1-D and 2-D computer simulations of MHD turbulence in molecular clouds.
\end{abstract}

Subject headings: hydromagnetics - ISM:clouds-ISM: kinematics and dynamics — ISM: magnetic fields - turbulence — waves

\section{INTRODUCTION}

Motions of material within interstellar molecular clouds have long been known to be dominantly turbulent except in small low-mass dense cores. A significant advance in the study of turbulence and cloud support was the finding of a set of scaling relations, i.e., 
correlations among cloud quantities such as the characteristic radius $R$, mean non-thermal velocity dispersion $\sigma_{v}$ and the average density $n_{H_{2}}=\rho / m$, for various samples of molecular structures ranging from $0.2 p c$ to $200 p c$ in size (Larson 1981; Leung et al 1982; Myers 1983; Dame et al 1986; Scoville \& Sanders 1987; Solomon et al 1987; Falgarone, Puget \& Perault 1992; Blitz 1993),

$$
\begin{gathered}
\sigma_{v} \propto R^{\alpha}, \alpha=0.5, \\
n_{H_{2}} \propto R^{-\gamma}, \gamma=1,
\end{gathered}
$$

and

$$
\sigma_{v} \propto n_{H_{2}}^{-\beta}, \beta=0.5
$$

Only two of the above relations are independent; the power-law indexes are related by $\alpha=\beta \gamma$. Since the above values for $\alpha, \beta$ and $\gamma$ are controversial, we will refer Equations (1)-(3) as the Standard Scaling Laws. Largely established on a cloud-cloud basis (i.e., based on samples of clouds), the physical significance of these scaling laws lies in the natural extrapolation that they are on average a manifestation of self-similar individual clouds satisfying these relations (Larson 1981), although arguably these correlations can be due to observational selection effects or even artifacts (Kegel 1989; Scalo 1990; Myers 1991; Vázquez-Semadeni et al 1996). In general, observational determination of these scaling relations is based on average properties of clouds and subject to numerous uncertainties. Therefore these scaling laws are necessarily rough and perhaps only of value dimensionally in a strict sense. On the other hand, the large number of observational studies that do find more or less the same scaling relations indicate that these scaling laws may indeed tell us something physical (Scalo 1987; McKee 1989), at least in a dimensional sense.

Proposed explanations to the scaling laws bifurcate into hydrodynamic and magnetohydrodynamic (MHD) regimes and were critically reviewed by Scalo (1987). Most of them converge on that one of the two independent scaling relations is due to the trend for virial equilibrium. The hydrodynamic approach takes the scaling laws as an evidence for energy cascade in strong eddy turbulence over a large range of scales, similar to the Kolmogorov law for incompressible turbulence (Larson 1981), which gives dimensionally $\sigma_{v} \sim R^{1 / 3}$. In the MHD regime, however, comparably rigorous theoretical approach has been hampered by the lack of theories capable of describing strong compressible MHD turbulence.

\section{EVIDENCE FOR A SCALE-INDEPENDENT FLUCTUATING MAGNETIC FIELD}


Given a relatively strong interstellar magnetic field and a moderate ionization fraction of $10^{-7}$ to $10^{-4}$ maintained by penetrating cosmic rays and UV photons, the gas in molecular clouds is expected to behave much like plasma which is subject to numerous instabilities. If one recognizes the existence of a general interstellar magnetic field and turbulence, then it seems difficult to reject the likelihood that the turbulent motions (even if it is initially purely hydrodynamic) further generate MHD waves such as Alfvén waves. In fact, Alfvén waves have long been proposed as a viable physical process for explaining the observed "turbulence" in molecular clouds (Arons and Max 1975, hereafter AM75; Zweibel \& Josafatsson 1983). Identifying (implicitly or explicitly) the turbulent velocity as the Alfvén velocity, it has been further proposed that the Standard Scaling Laws can be explained if kinetic energy, energy of the general magnetic field and gravitational energy are all in approximate equipartition and that magnetic field field $B$ is largely a constant over the scales concerned (Pellegatti Franco et al 1985; Scalo 1987; Myers \& Goodman 1988, hereafter MG88; Fleck 1988). The difficulty of understanding such a constant $B$, however, has been noted (Scalo 1987; MG88; Mouschovias \& Psaltis 1995); it contradicts not only the "frozen-in" picture for the interstellar magnetic field (cf. Mouschovias 1987) but also the observational evidence for a $B-n$ scaling relationship (Troland \& Heiles 1986; Heiles et al 1993).

The Doppler broadening of the spectral line profiles, nevertheless, reflects the velocity amplitudes of the waves (i.e. bulk moving velocity of the particles), not the phase velocity $V_{A}$. The velocity amplitude of an Alfvén wave packet of scale $\sim R$ is determined by the assumed equipartition between the kinetic energy density and the fluctuating magnetic energy density over the scale $R$ (AM75)

$$
\frac{1}{2} \rho \sigma_{v}^{2}=\frac{\sigma_{B}^{2}}{8 \pi} \text { or } \delta v \simeq \frac{\delta B}{(4 \pi \rho)^{1 / 2}},
$$

where $\delta v=(8 \ln 2)^{1 / 2} \sigma_{v}$ and $\delta B=(8 \ln 2)^{1 / 2} \sigma_{B}$. Alfvén (1953) was perhaps the first to apply this relation astrophysically (solar granulation). McKee \& Zweibel (1995) and Zweibel \& McKee (1995) have recently demonstrated this relationship for weak MHD turbulence (when $\delta B$ is much smaller than $B$ ). Although it is difficult to prove the validity of this relation in the strong compressible turbulence regime, both observational and theoretical studies of solar wind turbulence have confirmed this basic relation, which has become a basic building block of a lot of analyses (cf. Tu \& Marsch 1995; Lau \& Siregar 1996). Recent

\footnotetext{
${ }^{1}$ A Gaussian distribution is assumed for the fluctuating magnetic field and the velocity field with rootmean-squares $\sigma_{B}$ and $\sigma_{v}$. Note also that all the physical quantities in Equation (4) are implicitly associated with scale $R$.
} 
MHD turbulence simulations for molecular clouds also verified this energy equipartition (Passot et al 1995; Gammie \& Ostriker 1996).

Next, let us look at the commonly assumed virial equilibrium for gravitationally bound clouds. Observationally, virial equilibrium is established by comparing the kinetic and the gravitational energy of gas (cf. Larson 1981; Blitz 1993). Theoretically, however, the situation is much less trivial, as the presence of the general magnetic field should also be taken into account (cf. Nakano 1984; Mouschovias 1987; McKee et al 1995). Naively, the observationally established virial equilibrium seems to underscore the direct role of the general magnetic field, which is consistent with a possibility first raised by Mestel (1965) that the volume and surface terms due to the general magnetic field have opposite signs and thus tend to cancel. A potentially more interesting (and radical) possibility is that turbulent motions can effectively reduce the effective pressure or stress of the magnetic field (Kleeorin et al 1996). The gravitational energy density of a gas parcel at radius $R$ from the center of gravity can be written as $U_{G}=3 C G \frac{M(R) \rho}{R}$, where $C$ is a numerical factor depending mainly on the density distribution and $M(R)$ is the mass inside a radius $R$. The simplest form for the virial equilibrium in accord with observations can be written as

$$
C G \frac{M \rho}{R}=\rho \sigma_{v}^{2}+\frac{\rho k T}{m}
$$

where $m$ is mean mass of the molecules. This is the same approach as adopted by MG88 and Caselli \& Myers (1995). Defining a mean density $\rho_{m}$ so that $M(R)=\frac{4}{3} \pi \rho_{m} R^{3}$, the left hand side of Equation (5) becomes $C G \rho_{m} \rho R^{2}$. If $\rho_{m} \sim \rho$ within the errors of observational measurements, Equations (4) \& (5) lead to

$$
\rho=\left(\frac{4}{3} \pi C G\right)^{-1 / 2}\left(\frac{\sigma_{B}^{2}}{4 \pi}+\frac{\rho}{m} k T\right)^{1 / 2} R^{-1} .
$$

Equations (4) and (6) give

$$
\sigma_{v}=\left(\frac{4 \pi}{3} C G\right)^{1 / 4} \frac{\frac{\sigma_{B}}{(4 \pi)^{1 / 2}}}{\left(\frac{\sigma_{B}^{2}}{4 \pi}+\frac{\rho}{m} k T\right)^{1 / 4}} R^{0.5}
$$

It is clear that Equations (7),(6) and (4) match the Standard Scaling Laws, Equations (1), (2) and (3), respectively, if and only if $\sigma_{B}^{2}$ is scale-independent and thermal energy is small compared to the non-thermal energy. Since the turbulent pressure in this case is $P_{\text {turb }} \simeq \rho \sigma_{v}^{2}=\frac{\sigma_{B}^{2}}{4 \pi}$, a scale-independent $\sigma_{B}^{2}$ is consistent with the consensus that the scaling laws imply an approximate dynamic pressure equilibrium in molecular clouds (cf. Chièze 1987; Fleck 1988; Maloney 1988; Elmegreen 1989; Blitz 1993). For a sample of galactic molecular clouds associated with HII regions, Solomon et al (1987) found a 
velocity dispersion-size relationship $\sigma_{v}\left(k m s^{-1}\right)=0.7\left(\frac{R}{1 p c}\right)^{0.5}$. From Equation (7), we derive $\sigma_{B}=10.6 / C^{1 / 2} \mu$ gauss $=24 \mu$ gauss for $C \sim 0.2$ (uniform density). If these clouds are centrally condensed ( $C$ is somewhat larger), the required $\sigma_{B}$ will be correspondingly smaller. Note, however, when thermal kinetic energy density is not negligible on small scales, both the density-size and the velocity-size relations will be different from the standard forms. When thermal kinetic energy density dominates, Equation (6) yields the classic isothermal $r^{-2}$ density distribution; otherwise the power-law index $\gamma$ for the density-size relation ought to be between 1 and 2, as discussed by MG88, McKee (1989), Fuller \& Myers (1992) and Caselli \& Myers (1995).

\section{WAVE-WAVE INTERACTIONS: AN ANALOGY TO KOLMOGOROV CASCADE}

Since observations indicate that the turbulent kinetic energy is often comparable to the energy of the general background magnetic field in some molecular clouds (e.g., MG88; Crutcher et al 1993; 1994), MHD waves at different wavelengths are expected to have strong mutual interactions and thus become strong MHD turbulence (cf. Myers \& Khersonsky 1995). Intuitively, non-linear wave-wave interactions and consequent energy cascade largely dominate the spatial energy density distribution (cf. Sagdeev \& Galeev 1969; Elmegreen 1990; Biskamp 1994; Goldreich \& Sridhar 1995), similar to the Kolmogorov energy cascade for hydrodynamic eddy turbulence. Unfortunately, an eigenvalue description that is used for weak MHD turbulence breaks down in this case, leaving essentially no other effective means so far for establishing a working theory which could tell us what the spatial energy density distribution should be for strong compressible MHD turbulence. The theoretical difficulty is clearly witnessed by the recent controversy over the Iroshnikov-Kraichnan theory for incompressible MHD turbulence (e.g., Goldreich \& Sridhar 1995; Ng \& Bhattacharjee 1996), which is a much more simplified problem than strong compressible MHD turbulence in molecular clouds.

In this case it seems useful to see what observations tell us. Specifically, as argued in the previous section, the observed scaling laws in molecular clouds indicate a scale-independent fluctuating magnetic energy density $\sigma_{B}^{2}$. In terms of energy spectrum, $P_{k}$, i.e., energy per unit volume per unit wavenumber as a function of wavenumber $k \sim 1 / R$, a scale-independent

$\sigma_{B}^{2}$ implies a power-law $P_{k} \propto k^{-1}$. What is intriguing is that this $k^{-1}$ spectrum corresponds to equipartition of mode amplitudes (Biskamp 1994) or Raleigh-Jeans energy equipartition (Sagdeev \& Galeev 1969) in the sense that the wave packets of different scales obtain an 
equal share of the available energy within a unit volume of space. This implies further that wave packets on larger scales would dominate the total amount of energy.

Now, is such a spatial energy density distribution physically plausible ? We consider two aspects of the problem. First, the energy flux of an Alfvén wave packet on a scale

$R$ can be written as $S=V_{A} \frac{\sigma_{B}^{2}}{4 \pi}$ (cf. Lau \& Siregar 1996). It is evident that an ensemble of interacting wave packets traveling in all directions would yield a net energy transport flux $\Delta S$ only in the direction of the negative spatial gradient for the magnetic pressure or energy density $\sigma_{B}^{2} / 4 \pi$, and thus the developing tendency is to reduce the spatial gradients for $\sigma_{B}^{2}$. Second, a main characteristic of MHD turbulence is the existence of inverse cascade, i.e., energy cascade from small to large scales with the tendency to form self-organized large-scale coherent structures (Biskamp 1994), in contrast to the direct energy cascade (from large to small scales) in pure hydrodynamic turbulence. Therefore it is not unlikely that the interacting wave packets of different scales achieve a net tendency for an equal share of the available energy density due to the effective energy cascade in both the direct and inverse directions while the system is fully turbulent (cf. Biskamp 1994).

One point that appears interesting to us is that if the kinetic energy does remain in equipartition with the fluctuating magnetic energy, then perhaps a hydrodynamic description and a MHD description could lead to comparable physical insights to the turbulence in molecular clouds, at least from an energy point of view. The MHD approach, however, does offer certain advantages. One of these is that MHD waves may effectively transport energy with a high speed and perhaps also a low dissipation rate without necessary bulky flow of gas, and yet achieve more-or-less a uniform pressure environment. The disadvantage of the MHD approach is, however, its mathematical complexity.

Given that a scale-independent fluctuating magnetic field is physically plausible and the simplicity of it being able to explain the observed Standard Scaling Laws as discussed in previous section is remarkable, we feel obligated to propose this possibility as an empirical hypothesis for further observational and theoretical investigations.

\section{DISCUSSION}

\section{1. $\sigma_{B}$ versus $B$}

A fluctuating magnetic field component is not only implied by the observed "turbulence" or hydromagnetic waves (Alfvén 1953; AM75), but also expected to explain 
why molecular clouds do not free-fall to the center of mass along the generally-ordered magnetic field lines (cf. Shu et al 1987). Shu (1991) even suggested the intriguing possibility that an anisotropic fluctuating magnetic field might explain the reported prolateness of low mass cores. The fluctuating magnetic field $\sigma_{B}$ is, nevertheless, conceptually different from the general magnetic field $B$, even in the extreme case that $\sigma_{B} \sim B$. This raises the issue as for if the Standard Scaling Laws require a constant general magnetic field $B$ over a large range of scales, as some have discussed (e.g., Pellegatti Franco et al 1985; Scalo 1987; MG88; Fleck 1988). The answer to this question is negative based on the above discussions, but a constant $B$ would not contradict the scaling laws. A key point is that a constant $B$ does not directly explain the Standard Scaling Laws without invoking $\sigma_{B}$ first, because the Alfvén velocity is not the same as the observed fluctuating turbulent velocity amplitudes. In other words, even if $B$ is a constant over a large range of scales, a separate physical mechanism would still have to be sought to produce a scale-independent $\sigma_{B}$. In practice, however, it is likely that $\sigma_{B}$ becomes comparable to $B$ on large scales. From the empirical $B-n$ relation $B \simeq 1.5\left(\frac{n_{\mathrm{H}_{2}}}{1 \mathrm{~cm}^{-3}}\right)^{1 / 2} \mu$ gauss (Heiles et al 1993) and the estimated $\sigma_{B} \sim 24 \mu$ gauss (see Section 2 ), it can be crudely estimated that $\sigma_{B}$ becomes comparable to $B$ when $n_{H_{2}} \simeq 260 \mathrm{~cm}^{-3}, \sigma_{v} \simeq 2.3 \mathrm{~km} \mathrm{~s}^{-1}$ and $R \simeq 10 \mathrm{pc}$. Taking this at its face value, it seems that $\sigma_{B}$ could be well below $B$, or equivalently $\sigma_{v}$ considerably smaller than the Alfvén velocity on scales smaller than a few parsecs.

\subsection{Predictions}

Generally speaking, our principal expectation is that regions where the spatial distribution of the turbulent energy density is dominantly determined by wave-wave interactions are more likely to demonstrate the Standard Scaling Laws, while regions where this is not the case are unlikely to do so. Perhaps this is why the recent observational results (Plume et al 1996) of massive star forming cores do not agree with the standard scaling laws, as localized energy sources might dominate the energy density distributions over the scales of massive star formation. Further, the velocity-density relation (Equation (3)) among the Standard Scaling Laws should be the most robust physical relation in the sense that it may be expected for any region where magnetic fluctuations become scaleindependent. In contrast, the standard density-size and velocity-size relations require not only scale-independent magnetic field fluctuations, but also a second physical relationship 
such as the virial equilibrium?. This prediction seems supported by the observations of Falgarone, Puget \& Perault (1992) which find a remarkable correlation $\sigma_{v} \propto n_{H_{2}}^{-0.5}$ for a sample of gravitationally unbound molecular structures, although it is possible that jump shocks may also give rise to this relation (J.Scalo, private communication).

\subsection{Comparison with Existing 1-D and 2-D MHD Simulations}

Our hypothesis for a scale-independent fluctuating magnetic field, however, appears to contradict the recent 1-D and 2-D computer simulations for strong compressible MHD turbulence in molecular clouds. These simulations report a fluctuating magnetic spectrum (energy per unit volume per unit wavenumber), $P_{k} \propto k^{-t}$, where $t \sim 2.0-2.3$ for 1-D (Gammie \& Ostriker 1996) and $t \sim 1.85$ for 2-D (Passot, Vázquez-Semadeni \& Pouquet 1995). This magnetic spectrum implies a fluctuating magnetic field, $\sigma_{B} \propto R^{(t-1) / 2}$ (John Scalo, private communication). In other words, these simulations show a steeper k-dependence for the magnetic spectrum than our hypothesis. Both simulations find essentially the same velocity spectrum (energy per unit mass per unit wavenumber) $E_{k} \propto k^{-2}$, which is dimensionally consistent with the the observed standard velocity-size relation $\delta v \sim R^{1 / 2}$. It appears difficult for the simulations to explain the other observed Standard Scaling Laws. The difficulty of explaining the standard size-density relation in the 2-D simulations of Passot et al (1995), for example, is recently made clear by Vázquez-Semadeni et al (1996). These authors were then led to suggest that the standard velocity-size relation is the basic relation while the density-size relation may be an observational artifact, in clear contrast with our predictions in this paper. This raises the question as for if a simple dimensional analysis from a pure energy consideration as we have done in this paper is a valid approach for the MHD turbulence and if the equipartition between kinetic energy and fluctuating magnetic energy is a good assumption. On the other hand, 1-D and 2-D MHD turbulence simulations in solar wind studies have been known to produce energy spectra too steep to compare with observations, possibly due to the restriction in dimensionality, enforced periodicity and other unrealistic simplifications (Marsch 1991; Tu \& Marsch 1995). So it appears that the standard scaling laws and subsequently our hypothesis for a scale-independent fluctuating magnetic field in molecular clouds remain a challenge to MHD simulations, or vice versa. We share the same hope with

\footnotetext{
${ }^{2}$ Heithausen (1996) has recently reported a remarkable correlation $n \propto R^{-0.8}$ for high latitude clouds, which made him question if virial equilibrium is the true reason for a $n \propto R^{-1}$ relation. It is not clear yet, however, if his assuming equal distance for all the high latitude clouds would yield the correlations artificially.
} 
the solar physicists that future 3-D MHD simulations will shed light on this important issue in MHD turbulence.

In conclusion, we proposed the hypothesis that strong wave-wave interactions have led to a scale-independent distribution of fluctuating magnetic energy density and consequently the observed scaling laws in molecular clouds. We suggest that further careful observational studies of interstellar atomic/molecular clouds not in virial equilibrium or not harboring active star forming activities may serve as valuable direct tests of the basic ideas presented in this article, especially in comparison to the contradicting predictions of recent 1-D and 2-D computer simulations.

The author gratefully acknowledges kindest encouragement from Professors ChuanSheng Liu, Paul Goldsmith and Stuart Vogel. He is grateful to John Scalo for a critical review of the manuscript and numerous stimulating discussions. He further thanks Chuan-sheng Liu, John Wang, Frank Shu, Pedro Safier, Neal Evans, Paul Goldsmith, Phil Myers, Alyssa Goodman, Bruce Elmegreen, Dick Crutcher, Arieh Königl, Richard Larson, A. Bhattacharjee, Eve Ostriker and Enrique Vázquez-Semadeni for useful discussions. This research is supported in part by the NSF grant AST9314847 to the Laboratory for Millimeter-wave Astronomy at the University of Maryland.

\section{REFERENCES}

Alfvén, H. 1953, Cosmical Electrodynamics, (Oxford, The University Press), 146

Arons, J. \& Max, C.E. 1975, ApJ, 196, L77 (AM75)

Biskamp, D. 1994, Phys. Rev. E, 50, 2702

Blitz, L. 1993, in Protostars \& Planets III, ed. E.H. Levy \& J.I. Lunine (Tucson: The University of Arizona Press), 125

Caselli, P., \& Myers, P.C. 1995, ApJ, 446, 665

Chièze, J.-P. 1987; AA, 171, 225

Crutcher, R.M., Troland, T.H., Goodman, A.A., Heiles, C., Kazès, \& Myers, P.C. 1993, ApJ, 407, 175

Crutcher, R.M., Mouschovias, T.Ch., Troland, T.H., \& Ciolek, G.E. 1994, ApJ, 427, 839

Dame, T., Elmegreen, B., Cohen, R., \& Thaddeus, P. 1986, ApJ, 305, 892

Elmegreen, B.G. 1989, ApJ, 338, 178

Elmegreen, B.G. 1990, ApJ, 361, L77 
Falgarone, E., Puget, J.-L., \& Pérault, M. 1992, AA, 257, 715

Fleck, R.C. 1988, ApJ, 328, 299

Fuller, G.A., \& Myers, P.C. 1992, ApJ, 384, 523

Gammie, C.F., \& Ostriker, E.C. 1996, ApJ, 466, 814

Goldreich,P., \& Sridhar, S. 1995, ApJ, 438, 763

Heiles, C., Goodman, A.A., McKee, C.F., \& Zweibel, E.G. 1993, in Protostars \& Planets III, ed. E.H. Levy \& J.I. Lunine (Tucson: University of Arizona Press), 279

Heithausen, A. 1996, AA, 314, 251

Kegel, W.H. 1989, AA, 225, 517

Kleeorin, N., Mond, M., \& Rogachevskii, I. 1996, AA, 307, 293

Larson, R.B. 1981, MNRAS, 194, 809

Lau, Y.-T., \& Siregar, E. 1996, ApJ, 465, 451

Leung, C.M., Kutner, M.L., \& Mead, K.N. 1982, ApJ, 262, 583

Maloney,P. 1988, ApJ, 334, 761

Marsch, E. 1991, in Physics of the Inner Heliosphere: Particles, Waves and Turbulence, ed. R.Schwenn \& E. Marsch (New York: Springer), 159

McKee, C.F. 1989, ApJ, 345, 782

McKee, C.F., \& Zweibel, E.G. 1995, ApJ, 440, 686

McKee, C.F., Zweibel, E.G., Goodman, A.A. \& Heiles, C. 1993, in Protostars \& Planets III, ed. E.H. Levy \& J.I. Lunine (Tucson: University of Arizona Press), 327

Mestel, L. 1965, QJRAS, 6, 265

Mouschovias, T.Ch. 1987, in Physical Processes in Interstellar Clouds, ed. G.E.Morfill \& M. Scholer (Dordrecht:Reidel), 453

Mouschovias, T.Ch., \& Psaltis, D. 1995, ApJ, 444, L105

Myers, P.C. 1983, ApJ, 270, 105

Myers,P.C. 1991, in "Fragmentation of Molecular Clouds and Star Formation", ed. E.Falgarone \& G.Duvert (Dordrecht:Kluwer), 71

Myers, P.C., \& Goodman, A.A. 1988, ApJ, 329, 392 (MG88)

Myers, P.C., \& Khersonsky, V.K. 1995, ApJ, 442, 186

Nakano, T. 1984, Fundam. Cosmic Phys. 9, 139 
Ng, C.S., \& Bhattacharjee, A. 1996, ApJ, 465, 845

Passot, T., Vázquez-Semadeni, E., \& Pouquet, A. 1995, ApJ, 455, 536

Pellegatti Franco, G.A., Tarsia, R.D., \& Quiroga, R.J. 1985, ApSS, 111, 343

Sagdeev, R.Z., \& Galeev, A.A. 1969, Nonlinear Plasma Theory, (New York: W.A. Benjamin, Inc.)

Scalo, J.M. 1987, in Interstellar Processes, ed. D.J. Hollenbach \& H.A. Thronson, Jr. (Dordrecht:Reidel), 349

Scalo, J.M. 1990, in Physical Processes in Fragmentation and Star Formation, ed. R. Capuzzo-Dolcetta, C. Chiosi \& A. di Fazio (Dordrecht: Kluwer), 151

Scoville, N.Z. \& Sanders, D.B. 1987, in Interstellar Processes, ed. D.J.Hollenbach \& H.A. Thronson, Jr., (Dordrecht:Reidel), 21

Shu, F.H. 1991, in The Physics of Star Formation and Early Stellar Evolution, eds. C.J.Lada \& N.D.Kylafis (Dordrecht:Kluwer), 365

Shu, F.H., Adams, F.C., \& Lizano, S. 1987, ARAA, 25, 23

Solomon,P.M.,Rivolo,A.R.,Barrett,J.,\& Yahil, A. 1987, ApJ, 319, 730

Troland, T.H., \& Heiles, C. 1986, ApJ, 301, 339

Tu, C.-Y., \& Marsch, E. 1995, Sp. Sci. Rev., 73, 1

Vázquez-Semadeni, E., Ballesteros-Paredes, \& Rodr'iquez, L.F. 1996, ApJ, in press

Zweibel, E.G., \& Josafatsson, K. 1983, ApJ, 270, 511

Zweibel, E.G., \& McKee, C.F. 1995, ApJ, 439, 779 